\title{
Molding and Encoding Carbon Nitride-Containing Edible Oil Liquid Objects via Interfacial Toughening in Waterborne Systems
}

\author{
Qian Cao, Shahrouz Amini, Baris Kumru,* and Bernhard V. K. J. Schmidt* \\ Cite This: ACS Appl. Mater. Interfaces 2021, 13, 4643-4651 \\ Read Online
}

ABSTRACT: Charge interaction-driven jamming of nanoparticle monolayers at the oil-water interface can be employed as a method to mold liquids into tailored stable 3D liquid objects. Here, 3D liquid objects are fabricated via a combination of biocompatible aqueous poly(vinyl sulfonic acid, sodium salt) solution and a colloidal dispersion of highly fluorescent organomodified graphitic carbon nitride $\left(\mathrm{g}-\mathrm{C}_{3} \mathrm{~N}_{4}\right)$ in edible sunflower oil. The as-formed liquid object shows stability in a broad $\mathrm{pH}$ range, as

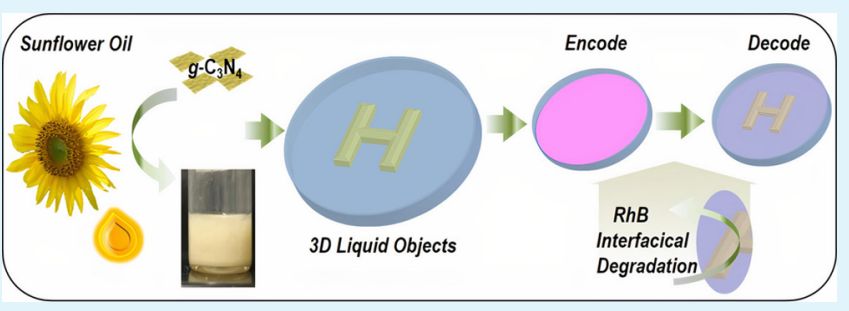
well as flexible pathways for efficient exchange of molecules at the liquid-liquid interphase, which allows for photodegradation of rhodamine $\mathrm{B}$ at the interface via visible light irradiation that also enables an encoding concept. The $\mathrm{g}-\mathrm{C}_{3} \mathrm{~N}_{4}$-based liquid objects point toward various applications, for example, all-liquid biphasic photocatalysis, artificial compartmentalized systems, liquid-liquid printing, or bioprinting.

KEYWORDS: aqueous liquid object, carbon nitride, interface, liquid printing, photocatalysis

\section{INTRODUCTION}

Soft water-based materials have found various areas of utilization because of their favorable interactions with biological entities, while providing macroscopic flexibility and microscopic molecular mass transport. ${ }^{1-3}$ Therefore, a broad range of applications in biological systems is in the focus of research, such as tissue engineering, ${ }^{4}$ drug delivery, ${ }^{5}$ or selfhealing. ${ }^{6,7}$ Another direction is the implementation of multiphase liquid systems that enable the formation of compartmentalized soft materials ${ }^{8,9}$ but give access to other applications like catalysis, ${ }^{10}$ particle separation, ${ }^{11}$ and sensing ${ }^{12}$ as well. A recent development in soft materials is the introduction of structured liquids, which is based on nanostructured assemblies at the interphase of biphasic liquid media to generate mechanically stable interphases. ${ }^{2}$ Interfacial jamming occurs between two liquid phases during the process of decreasing the interfacial area while minimizing the interfacial energy. At the same time, the binding energy increases, which is different from typical surfactants or Janus systems. Finally, an interfacial layer or film structure is obtained that is strong and robust against stress from the direction of both phases. Recently, these structured liquids have attracted growing interest because of their unique structural and physicochemical properties. ${ }^{13,14}$

The fabrication of liquid objects via jamming between two liquid phases has been described recently by Russell and coworkers, who are pioneers in this field. ${ }^{15-20}$ Interactions between functionalized polymers dissolved in one phase (mostly organic solvent) and nanoparticles dispersed in an aqueous system were utilized to enable electrostatic or noncovalent interaction forming solidified interfaces in a supramolecular approach. For example, interfacial jamming of cellulose nanocrystals and amine-terminated poly(styrene) at a water-carbon tetrachloride interface was introduced. ${ }^{21}$ In other works, shape-controllable liquid objects that respond to external magnetic stimuli can be fabricated by a combination of magnetic nanoparticles with amine-modified polyhedral oligomeric silsesquioxane at the water-toluene interface. ${ }^{22}$ Photoresponsive structured liquids can be realized by hostguest molecular recognition as well. ${ }^{23}$ Recently, the liquid structuring technology was applied for the construction of DNA microcapsules. ${ }^{24}$ One of the main advantages of liquid structuring is the formation of a flexible but stable interface that can be tailored via several parameters, like polymer molar mass, ${ }^{25} \mathrm{pH}$ of the aqueous phase, ${ }^{26}$ and size or concentration of nanoparticles. ${ }^{22}$

So far, mainly the organic phase was utilized as the continuous phase in liquid structuring, relying on relatively expensive charged polymers as ligands, while aqueous-based systems are rarely reported. ${ }^{2}$ Regarding the state-of-the-art systems, volatile organic solvents (e.g., toluene ${ }^{16,27}$ ) and nonvolatile organic oils (e.g., silicone oil ${ }^{28}$ ) were successfully applied to construct the structures, whereas long-term stability,

Received: October 8, 2020

Accepted: January 8, 2021

Published: January 19, 2021 
safety features, and scalability of the structured liquid systems were addressed scarcely. Especially, the implementation of toxic organic solvents restricts further applications in biorelated fields. Thus, development of nontoxic and biocompatible liquid systems with water as the continuous phase is of significant interest.

Graphitic carbon nitride $\left(\mathrm{g}-\mathrm{C}_{3} \mathrm{~N}_{4}\right)$ has attracted extensive attention in the past decade, which is due to its performance as a metal-free heterogeneous photocatalyst with adjustable optical and physicochemical properties. ${ }^{29-34}$ The photocatalytic properties of $\mathrm{g}-\mathrm{C}_{3} \mathrm{~N}_{4}$ have been exploited for a variety of applications including photodegradation for waste-water treatment, ${ }^{35} \mathrm{CO}_{2}$ reduction, ${ }^{36,37}$ water splitting, ${ }^{38}$ in combination with polymers, ${ }^{39,40}$ polymerization catalysis, ${ }^{41}$ and the fabrication of hydrogels. ${ }^{42,43}$ Besides photocatalytic properties of $\mathrm{g}-\mathrm{C}_{3} \mathrm{~N}_{4}$, surface and colloidal properties have been of interest as well, ${ }^{44}$ for example, in the role of a Pickering emulsion stabilizer for organic/water emulsions ${ }^{45,46}$ and in emulsion polymerization processes. ${ }^{47,48}$ Furthermore, the tailoring of $\mathrm{g}-\mathrm{C}_{3} \mathrm{~N}_{4}$ surface functionality by photoinduced modification enabled integration into an organic/oil phase rather than at the interface. ${ }^{49,50}$ Subsequently, colloidal properties have come into focus of research in order to overcome low dispersibility in liquid media. ${ }^{39,51,52}$ Recently, a vinylthiazole-functionalized phenyl-modified carbon nitride (vTA-CMp) was reported, which showed considerable organo-dispersibility, high colloidal stability, and strong fluorescent emission. ${ }^{53}$ Meanwhile, vTA-CMp exhibits electrostatic stabilization due to migration of negative charges to the thiazole edges and positive charges toward the inner part of phenyl-modified carbon nitride (CMp) sheets when dispersed in organic solvents, ${ }^{53}$ for example, in $N$-methyl pyrrolidone or isopropyl alcohol.

Inspired by these facts, we introduce liquid structuring based on vTA-CMp. Therefore, organo-dispersible vTA-CMp is dispersed in edible sunflower oil to act as the organic phase (Scheme 1a), which has to the best of our knowledge never been introduced as an organic fluid component in liquid structuring. Aqueous poly(vinyl sulfonic acid, sodium salt) (PVSA) solution with negatively charged sulfonate groups constitutes the outer phase. A facile and versatile method is introduced to form molded yet all-liquid objects by supramolecular interaction (Scheme 1b) that feature shape

Scheme 1. (a) Dispersion of vTA-CMp in Sunflower Oil; (b) Process of Molding Liquid Objects: (A) Prewetting the Mold with PVSA Solution, (B) Filling the Mold with vTACMp/Oil, (C) Filling the Whole Surrounding with Aqueous PVSA Solution, and (D) Lifting the Mold to Receive the Liquid Object; and (c) Application of vTA-CMp Liquid Objects to Encode and Decode via Photocatalysis

(a)

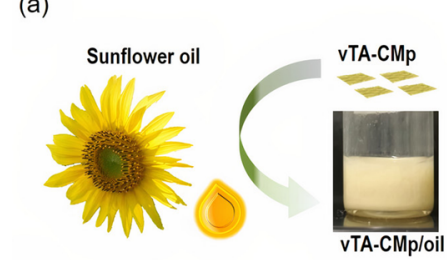

(b)

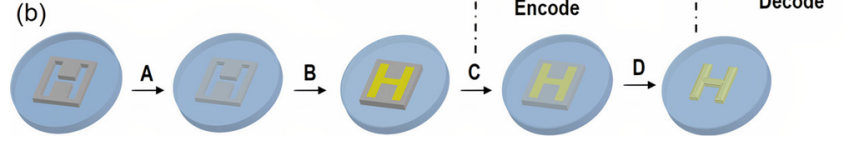

persistence over a wide $\mathrm{pH}$ range. To understand the structure formation and its stability, the interfacial behavior and the mechanism of interfacial film formation between vTA-CMp/oil and aqueous PVSA solution are investigated. The vTA-CMp liquid object exhibits potential utilization to encode information in liquid media that can be decoded under external stimuli (Scheme 1c), utilizing the photoactivity of vTA-CMp. Although Pickering stabilization of oil-in-water emulsions with $g-C_{3} N_{4}$ has been described in the literature, the present system introduces a new level of liquid structuring via g- $\mathrm{C}_{3} \mathrm{~N}_{4}$ mediation that features photocatalytic properties at the same time.

\section{EXPERIMENTAL SECTION}

Materials. Cyanuric acid (98\%, Sigma-Aldrich), 2,4-diamino-6phenyl-1,3,5-triazine (97\%, Sigma-Aldrich), hexadecyltrimethylammonium bromide (CTAB, $\geq 99 \%$, Sigma-Aldrich), hydrochloric acid ( $\mathrm{HCl}$, fuming, Carl Roth), melamine (99\%, Sigma-Aldrich), 4-methyl5-vinylthiazole (vTA, 97\%, Sigma-Aldrich), rhodamine B (RhB, Sigma-Aldrich), sodium hydroxide $(\mathrm{NaOH} ; 98 \%$, Sigma-Aldrich), poly(styrene sulfonic acid, sodium salt) (PSSA, $M_{\mathrm{w}} 4300 \mathrm{~g} \mathrm{~mol}^{-1}$ Sigma-Aldrich), and PVSA solution ( $30 \mathrm{wt} \%$ in $\mathrm{H}_{2} \mathrm{O}$, Sigma-Aldrich, $M_{\mathrm{w}} 2500 \mathrm{~g} \mathrm{~mol}^{-1}$ ) (Figure S1) were used as purchased. Sunflower oil was purchased from the supermarket and used without further purification. The homemade polyamide (PA) " $\mathrm{H}$ " mold was designed using Autodesk Inventor Professional 2020 and manufactured with a CNC milling machine (Germany). $50 \mathrm{~W}$ light-emitting diode (LED) chips (Bridgelux BXRA-50C5300; $\lambda>410 \mathrm{~nm}$ ) were connected to a self-made circuit and cooling system. CMp and vTA-CMp were synthesized according to the literature (refer to the Supporting Information). ${ }^{53,54}$

Liquid Object Preparation. $20 \mathrm{mg}$ of the as-prepared vTA-CMp was added to $1 \mathrm{~mL}$ of sunflower oil and ultrasonicated for $1 \mathrm{~h}$ to obtain vTA-CMp/oil dispersion, followed by centrifugation ( $500 \mathrm{rpm}$, $3 \mathrm{~min}$ ) to remove the residual aggregates via sedimentation, and finally, a colloidal vTA-CMp/oil dispersion with a concentration of 15 $\mathrm{mg} \mathrm{mL} \mathrm{m}^{-1}$ was obtained (concentration measured gravimetrically). The " $\mathrm{H}$ " mold was first put in an empty Petri dish, and both the dish and the mold were prewetted with PVSA solution (ca. $100 \mu \mathrm{L}$ ), then the mold was filled with vTA-CMp/oil $(400 \mu \mathrm{L})$, and then the top of the oil phase was covered with PVSA solution. Finally, the whole Petri dish was filled with aqueous PVSA solution. After $10 \mathrm{~min}$, the " $\mathrm{H}$ " mold was slowly taken out of the solution, leaving the formed liquid object with "H" shape behind. The original PVSA solution possesses a $\mathrm{pH}$ value of 7; for investigating acidic and alkaline environments for the formation of the liquid " $\mathrm{H}$ " object, PVSA solution with $\mathrm{pH}$ values of 2 and 13 were adjusted by adding $0.01 \mathrm{M}$ of $\mathrm{HCl}$ or $\mathrm{NaOH}$ solution, respectively. The remaining procedure was kept the same as described above.

RhB Photodegradation. The liquid letter sample for RhB degradation was obtained with the same procedure as described above. In addition to the mentioned reagents, $\mathrm{RhB}$ was added to the aqueous PVSA solution (with specific concentrations that are mentioned in the result and discussion) before the final step of filling the Petri dish. After the formation of the " $\mathrm{H}$ " liquid object and removal of the mold, a $50 \mathrm{~W}$ LED chip was placed above the sample with a distance of $20 \mathrm{~cm}$ and irradiation was started. Samples and the corresponding images were collected after certain time intervals. The $\mathrm{RhB}$ concentration was monitored spectrophotometrically utilizing its absorption maximum $(\lambda=554 \mathrm{~nm})$.

Characterization. Molar mass of PVSA was measured in aqueous $0.1 \mathrm{M} \mathrm{NaNO}_{3}$ buffer by size exclusion chromatography (SEC) using PSS 1260-Iso as the pump, an anionic column system with PSSSECcurity-UV and PSS-SECcurity-RI as detectors, and a calibration with PSS $\mathrm{Na}$ as standard.

The depth-sensing indentation measurements were performed using a Triboindenter TI-950 (Hysitron-Bruker, MN, USA) equipped with an extended displacement stage, allowing for maximum 
displacements of up to $500 \mu \mathrm{m}$. Two different type of tips were used in this work, a fluid-cell diamond tip with a nominal radius of $50 \mu \mathrm{m}$ and a customized glass tip with a radius of $425 \mu \mathrm{m}$ were used for "airindent" measurements and fluid resistance measurements, respectively. The first series of the experiments were conducted using an "air-indent" method ${ }^{55}$ on the single liquid phases and while the tip was initially away from the surface of the liquid. Accordingly, over a large displacement loading and unloading, the "jump-in" and capillary bridging events were recorded. In the second series of the measurements, a cono-spherical glass tip (Figure S2) was used for probing because of the intolerance of the standard diamond tip in an acidic or basic environment. To improve recording of the fluid resistance and the tip-interface interactions, the experiments were performed while the glass tip was immersed inside the liquid phases. As the interfacial jamming is non-uniform around the object, all-round probing was conducted, and the tested area is marked in Figure S3.

The interfacial tension of aqueous PVSA solutions with different $\mathrm{pH}$ values was determined by pendant drop analysis with an optical contact angle instrument (Dataphysics ES, Germany). The pendant drop tensiometer was used to measure the interfacial tensions of the oil and water interface with varying $\mathrm{pH}$ of vTA-CMp/PVSA films. 2 $\mathrm{mL}$ of sunflower oil or vTA-CMp containing sunflower oil were poured into a cuvette and a volume of ca. $30 \mu \mathrm{L}$ water or aqueous PVSA solution was slowly injected by a syringe. Then, the droplet shape profile was analyzed and the pressure difference between oil and water fluids was calculated by the Young-Laplace equation. For each sample, at least three independent measurements were performed. The dynamic interfacial tension was recorded after the aqueous PVSA solution droplet was slowly injected into the vTA-CMp/oil phase, and the droplet morphology and deformation were recorded by a camera provided by the equipment manufacturer. The particle size of vTA$\mathrm{CMp}$ nanosheets and zeta potential was determined using a Zeta Nanosizer instrument (Malvern Instruments, UK) at a fixed scattering angle of $90^{\circ}$. Charges of vTA-CMp dispersion in oil were measured with a Mütek PCD 03 particle charge detector.

\section{RESULTS AND DISCUSSION}

Liquid Object Formation. Organo-dispersible vTA-CMp (Figure S4a) was formed by photofunctionalization of CMp (Figure S4b) via vTA following our previous work. ${ }^{53}$ For the first time, edible sunflower oil was introduced as a benign dispersion medium for $\mathrm{g}-\mathrm{C}_{3} \mathrm{~N}_{4}$. A colloidal vTA-CMp/oil dispersion with vTA-CMp nanosheets with an average size of $290 \mathrm{~nm}$, as measured via dynamic light scattering (Figure S4e), was obtained by ultrasonication and centrifugation (Scheme 1a). It is anticipated that CMp possesses negative surface charge randomly located around defects, which supports colloidal stabilization. ${ }^{54}$ Furthermore, CMp possesses smaller sheet size and phenyl decorated edges compared to traditional g- $\mathrm{C}_{3} \mathrm{~N}_{4} \mathrm{~s},{ }^{54}$ which facilitates the exfoliating process and further molecular modification by aromatic vTA molecules. After covalent modification with the vTA group, the modified g$\mathrm{C}_{3} \mathrm{~N}_{4}$ vTA-CMp gains excellent colloidal stability. Modification with vTA not only provides enhanced organo-dispersibility, but also induces migration of negative charges toward modified edges, while the CMp core remains positively charged. ${ }^{53}$ Such a polarized structure endows vTA-CMp with excellent electrostatic stabilization, even in organic solvents as reported previously. ${ }^{53}$ Regarding these properties, electrostatic interactions between polarized vTA-CMp and polyelectrolytes in a liquid-liquid heterophase system would enable moldable liquid objects if adjusted properly that can be also a direction of new systems for liquid-liquid printing. For example, PVSA can be introduced, which is a biocompatible polymer with negatively charged sulfonate groups in an aqueous environment. ${ }^{56} \mathrm{~A}$ homemade mold from hydrophobic PA with a patterned bottom open trench in the shape of the letter "H" was manufactured by a milling machine (Figure S5), which has a contact angle with PVSA solution and vTA-CMp/oil of 42.3 and $16.9^{\circ}$, respectively (Figure S6a,b). Prior to all-liquid molding, both the Petri dish and mold were prewetted with aqueous PVSA solution (30 wt \%). The prewetting process is necessary, otherwise the vTA-CMp/oil dispersion can stick to the corners because of the small contact angle with PA and influence the final shape of the liquid object. Subsequently, vTA-CMp/oil dispersion (with a concentration of $15 \mathrm{mg} \mathrm{mL}^{-1}$ as prepared) was added to the prewetted trench and capped with aqueous PVSA solution to form the interfacial layer. Then, the whole Petri was filled with aqueous PVSA solution until the mold was completely immersed. After $10 \mathrm{~min}$, the mold was removed and the presence of interfacial electrostatic interactions between vTA-CMp nanosheets with PVSA prevented the collapse of the "H"-shaped vTA-CMp/oil phase, thanks to interfacial film formation. Even though PVSA solution with a density of $1.23 \mathrm{~g} \mathrm{~cm}^{-3}$ is denser than vTA-CMp/oil $\left(0.92 \mathrm{~g} \mathrm{~cm}^{-3}\right)$ (Table S1), the liquid "H" structure rested at the bottom of the PVSA solution $(\mathrm{pH}=7$, Figure 1a). As vTA-CMp possesses excellent fluorescent
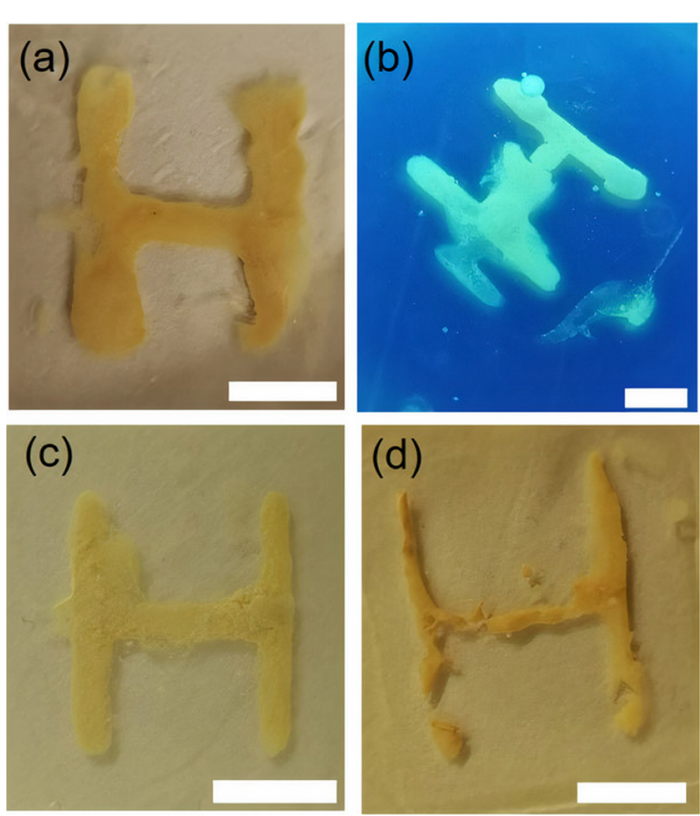

Figure 1. (a) Molded liquid letter "H" in a vTA-CMp/oil (15 mg $\left.\mathrm{mL}^{-1}\right)$ and aqueous PVSA solution (30 wt $\%$ in water) system $(\mathrm{pH}=$ 7). (b) Fluorescent liquid object " $\mathrm{H}$ " under UV light irradiation at a wavelength of $365 \mathrm{~nm}$. (c,d) "H" letter constructed in a vTA-CMp/oil $\left(15 \mathrm{mg} \mathrm{mL}^{-1}\right.$ ) and aqueous PVSA solution ( $30 \mathrm{wt} \%$ in water) system with adjusted $\mathrm{pH}$ of PVSA solution of 2 and 13, respectively. The scale bar in the images represents $50 \mathrm{~mm}$.

properties, the liquid object " $\mathrm{H}$ " features green light emission under UV light irradiation (Figure 1b). Moreover, the liquid objects tolerate strong acidic conditions (Figure 1c). In an alkaline environment, less stable structures, that is, thinner liquid architectures were formed at $\mathrm{pH} 13$ (Figure 1d). Lower concentrations of vTA-CMp/oil $\left(5 \mathrm{mg} \mathrm{mL}^{-1}\right)$ and PVSA (15 wt $\%$ in $\mathrm{H}_{2} \mathrm{O}$ ) were also studied (Figure S7) and complete liquid structures could be formed in both experiments. This implies that for further applications, especially biorelated ones, utilization of lower concentrations is possible for a stable liquid object formation. 

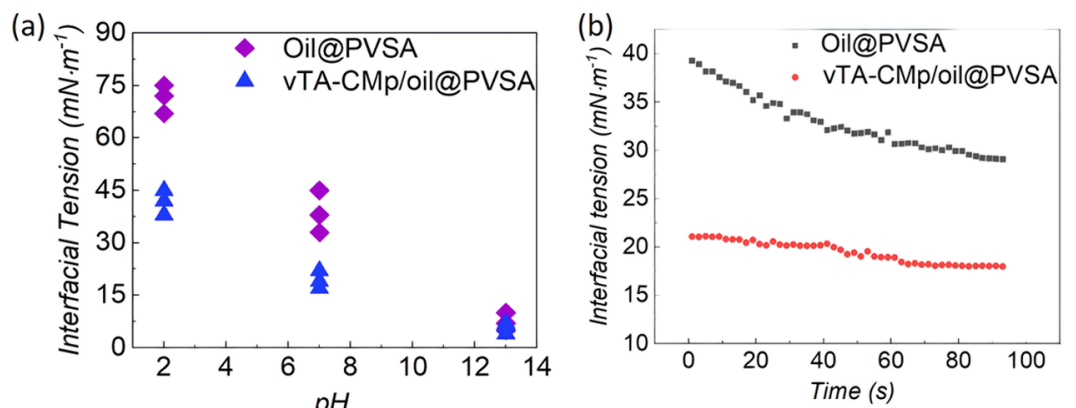

(e)

(c)

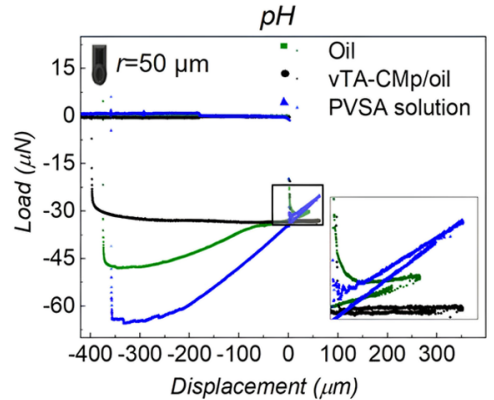

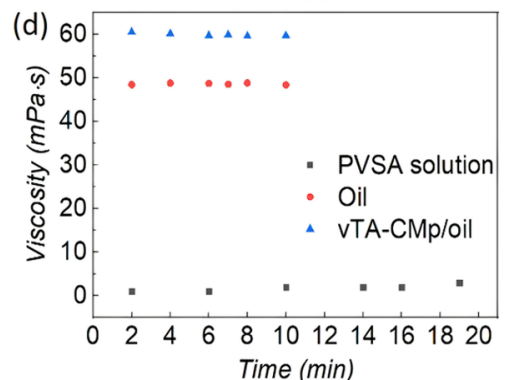

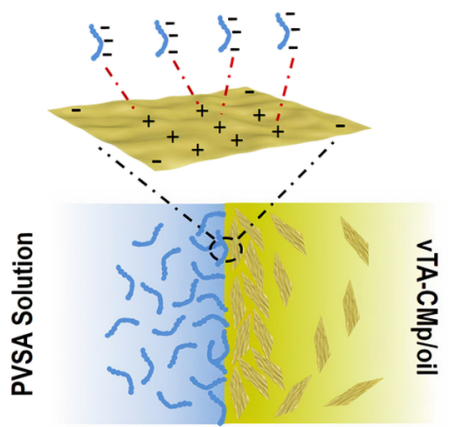

Figure 2. (a) Interfacial tension determined by a pendant drop of the vTA-CMp/oil and aqueous PVSA solution interface with different $\mathrm{pH}$, three independent experiments for each condition. (b) Equilibrium observation of interfacial tension of vTA-CMp/oil and aqueous PVSA solution at pH = 7. (c) "Air-indent" of the contact force with depth sensing of oil, vTA-CMp/oil, and aqueous PVSA solution with a diamond tip with a nominal radius of $50 \mu \mathrm{m}$. (d) Dynamic viscosity of oil, vTA-CMp/oil, and aqueous PVSA solution measured with a micro viscometer at $25^{\circ} \mathrm{C}$. (e) Cartoon illustration of the interaction between vTA-CMp and PVSA polymer chain at sunflower oil and water interface.

Liquid Object Interfacial Properties. On the one hand, the interfacial properties of the formed liquid objects are of particular interest for future applications in multicompartment systems. On the other hand, to understand the formation of the liquid object and its stability, interfacial properties are the key. Therefore, the interfacial behavior between PVSA and vTA-CMp with respect to $\mathrm{pH}$ was studied by interfacial tension $(\gamma)$ measurements (Figure 2a) using a pendant drop of an aqueous PVSA solution droplet in vTA-CMp/oil dispersion. In contrast to the literature, ${ }^{22}$ no obvious wrinkling and deformation was observed with pendant drop measurements. The $\gamma$ value between oil and aqueous PVSA solution is $70 \pm 4$, $38 \pm 5$, and $7 \pm 3 \mathrm{mN} \mathrm{m}^{-1}$ with increasing $\mathrm{pH}$ values of 2,7 , and 13 , respectively, which indicates interfacial stability of PVSA over the $\mathrm{pH}$ region studied. By dispersing vTA-CMp in oil, the interfacial tension between aqueous PVSA solution and vTA-CMp/oil decreased significantly to $41 \pm 4,17 \pm 3$, and 5 $\pm 2 \mathrm{mN} \mathrm{m}^{-1}$ corresponding to increasing $\mathrm{pH}$ compared to pure oil. The decrease in $\gamma$ indicates adsorption of vTA-CMp at the oil/water interface, which is beneficial for minimizing the interfacial energy and formation of a stable interfacial film. As CMp is a sheetlike robust material, the aggregation of vTA$\mathrm{CMp}$ at the interface boosts the interfacial mechanical properties and is beneficial for maintaining the integrity of the interfacial layer. Overall, $\gamma$ of the two phases decreased with the increasing $\mathrm{pH}$ value from 2 to 7 and 13 . As the sulfonic acid group is a $\mathrm{pH}$-sensitive group, changing $\mathrm{pH}$ alters the properties of the PVSA polymer chain as well as chain structure in aqueous solution. Another issue is that the changing $\mathrm{pH}$ also affects the net surface charge of vTA-CMp at the liquid-liquid interface. To elucidate the corresponding $\mathrm{pH}$ to the point of zero charge for vTA-CMp, zeta potential was measured with varying $\mathrm{pH}$ values in water, indicating that the isoelectric point of vTA-CMp is around the $\mathrm{pH}$ value of 5 (Figure S8). The surface charge of vTA-CMp in oil is $+7 \mathrm{mV}$, as measured with a particle charge detector.
At a $\mathrm{pH}$ value of 2, the sulfonic acid group is protonated to some extent and less amount of the charged sulfonic group is left compared to more basic $\mathrm{pH}$. At the $\mathrm{pH}$ values of 7 and 13 , the PVSA chains will be deprotonated. Additionally, at the $\mathrm{pH}$ value of 2, vTA-CMp possesses a zeta potential around +19 $\mathrm{mV}$ in water, indicating not only sulfonic acid groups, but also vTA-CMp at the liquid-liquid interface can be protonated leading to weakened interactions. This ultimately leads to a less stable assembly at the interface and thus to increased $\gamma$. For higher $\mathrm{pH}(\mathrm{pH}=13)$, the interfacial tension was minimized with or without vTA-CMp, which is directly related to a stable interfacial area in both cases. Thus, the aggregation of vTA$\mathrm{CMp}$ at the interface is not required to minimize the interfacial tension at high $\mathrm{pH}$. However, as vTA-CMp introduces an increased mechanical stability to the interfacial elastic film, the presence of vTA-CMp nanosheets is required to maintain the shape of nonequilibrium liquid objects. With an addition of $\mathrm{NaOH}$, the formation of a highly negative surface charge of vTA-CMp in water indicates that vTA-CMp also interacts with $\mathrm{OH}^{-}$in aqueous solution. Importantly, the sulfonic acid group is deprotonated and the $\mathrm{OH}^{-}$-rich environment resulted in significant repulsion of $\mathrm{OH}^{-}$with the sulfonic acid group, causing precipitation of polymer chains in the aqueous phase; hence, polymer chains tend to aggregate in the interfacial area and lower the interfacial force to maintain the stability. However, the aggregation at the interfacial area also led to a volume pressure to the oil phase, which causes extrusion of oil from the vTA-CMp/oil phase, and only a thinner and noncontinuous liquid object was formed.

At a $\mathrm{pH}$ value of 7 , the interfacial tension between pure oil and water is $208 \mathrm{mN} \mathrm{m}^{-1}$, with a persistence time of $300 \mathrm{~s}$ (Figure S9). Addition of PVSA in water effectively decreased the interfacial tension $\left(33 \mathrm{mN} \mathrm{m}{ }^{-1}\right)$ with shorter persistence time of $60 \mathrm{~s}$ only (Figure S10), which indicates that PVSA is beneficial for the formation of a lower energy interfacial area between oil-water phases. The addition of vTA-CMp to the 

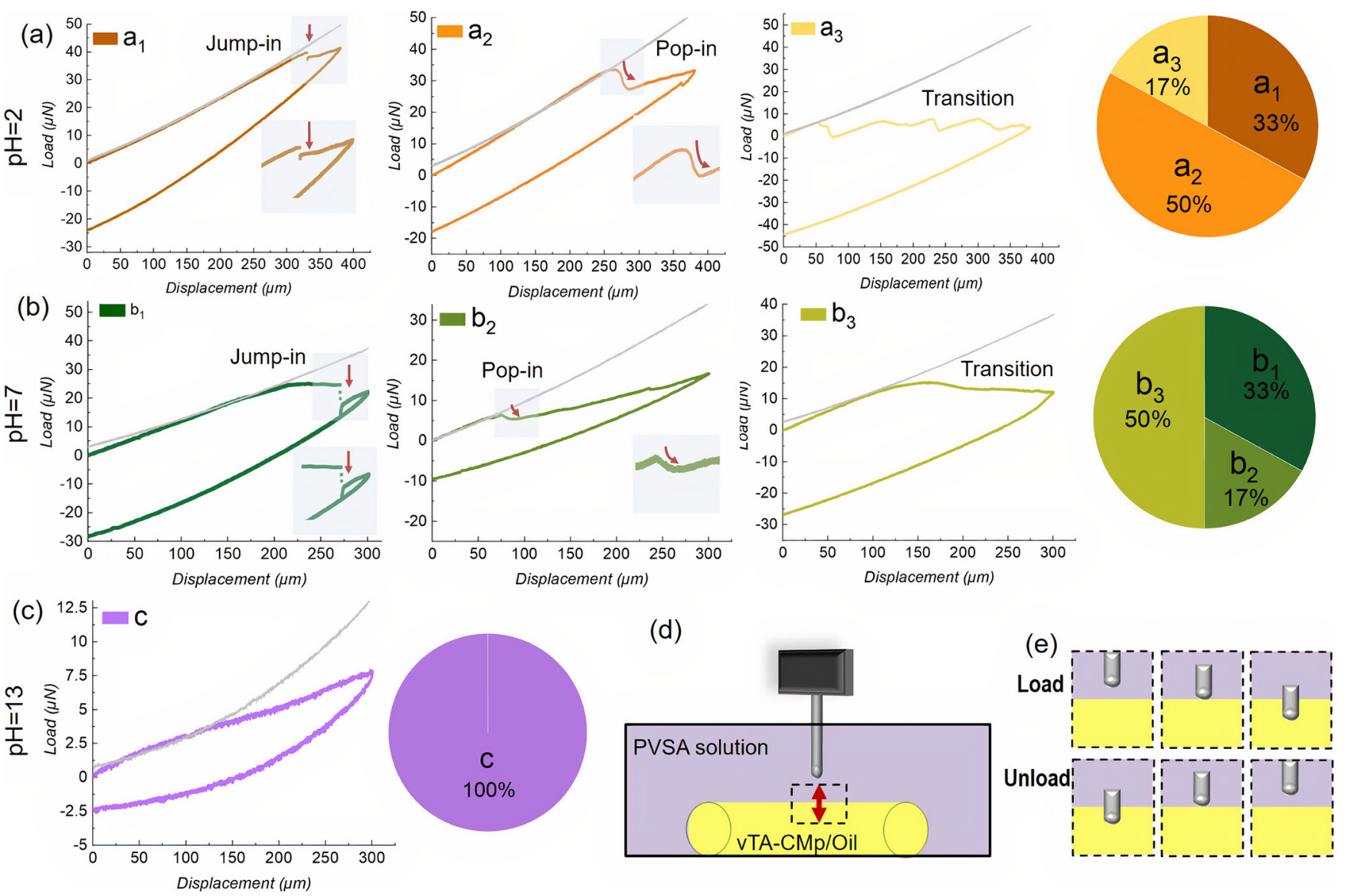

Figure 3. Fluid resistance curves extracted from depth-sensing indentations with a cono-spherical glass tip $(r=425 \mu \mathrm{m})$ approaching the vTA$\mathrm{CMp} /$ oil-PVSA solution interfacial area with different $\mathrm{pH}$ values of PVSA solution: (a) $\mathrm{pH}=2,(\mathrm{~b}) \mathrm{pH}=7$, and (c) $\mathrm{pH}=13$ (the gray curve in each graph represents the depth-sensing indentations of PVSA solution in the corresponding $\mathrm{pH}$ value. Deviation from this curve denoting the interaction of the tip with the interface). (d) Illustration of the fluid resistance measurements using a cono-spherical glass tip with a radius of 425 $\mu \mathrm{m}$ and the probing area is highlighted with a dashed box. (e) Illustration of the load and unload process of the probing process which is highlighted with a dashed box in (d).

oil phase also effectively decreased the interfacial energy, as interfacial tension between vTA-CMp/oil and pure water is 74 $\mathrm{mN} \mathrm{m}^{-1}$ with a persistence time of $340 \mathrm{~s}$ (Figure S11). The longer persistence time compared to the PVSA solution-pure oil system is due to the fact that vTA-CMp acts as a surfactant and as an interfacial reinforcer. Moreover, the interfacial tension between vTA-CMp/oil and PVSA solution is stable over an observation time of $90 \mathrm{~s}$, while pure oil and aqueous PVSA solution show gradually decreasing interfacial tension over an observation time of $90 \mathrm{~s}$ (Figure $2 \mathrm{~b}$ ), which indicates the stable interfacial area formation with vTA-CMp/PVSA interfacial assembly. At a $\mathrm{pH}$ of 7, the PVSA solution-based droplet remains stable in the vTA-CMp/oil dispersion with a duration of at least $40 \mathrm{~min}$ (Figure S12), and the enhanced persistence time of the PVSA droplet can be ascribed to the interaction of vTA-CMp nanosheets and PVSA polymer chain. Hence, an interfacial elastic film is formed that maintains the position of the PVSA droplet and prolongs the time of droplet stability. With changing the $\mathrm{pH}$ of PVSA solution to 2 and 13, persistence times of $80 \mathrm{~s}$ (Figure S13) and $0 \mathrm{~s}$ were observed, respectively. As mentioned before, at $\mathrm{pH}$ of 2 , the sulfonic acid group is partially protonated, leading to less interfacial assembly, which resulted in a shorter persistence time similar to the PVSA-oil system. For a $\mathrm{pH}$ of 13 , high volume pressure that resulted from the aggregation of the PVSA polymer chain led to an instant dropdown of the PVSA droplet in the vTA$\mathrm{CMp} /$ oil phase.

The interface structure was further assessed via light microscopy imaging of liquid objects, showing obvious aggregation of vTA-CMp nanosheets at the interface (Figure S14). As mentioned before, vTA-CMp possesses excellent electrostatic stabilization properties due to the polarized structure, which may contribute to electrostatic interaction with negatively charged PVSA and thus stabilization of the interfacial film (Figure 2e).

Indentation Studies. To improve the understanding of the interfacial interaction of the liquid phases, we investigated the adhesion properties of the individual liquid phases using the "air-indent" depth-sensing technique first. ${ }^{55}$ In this technique, the interactions of a cono-spherical diamond indenter $(r=50 \mu \mathrm{m})$ and a liquid phase can be recorded in a cycle of approaching to, interacting with, and retracting from the surface of a liquid over a $500 \mu \mathrm{m}$ displacement (Figure S15). Accordingly, the technique enables detecting the jump-in event, while approaching and record capillary bridging over retracting, which can be used to extract the fluid resistance force and adhesion force of the liquid phase, respectively (Figure 2c). As the diamond probe approaches the liquid surface while loading, the surface energy of the liquid retracts the tip toward the liquid surface resulting in a jump-in event. 
Right after the jump-in event, the fluid resistance results in an increment in the load. By a rough comparison of the load increment rates (Figure 2c, inset, the slope of the curves after the jump-in events), the PVSA solution shows a higher resistance than oil. This response was followed by a higher adhesion force $(65 \pm 2 \mu \mathrm{N}$ with a length of $\sim 350 \mu \mathrm{m})$ over retraction of the indenter from the surface of the PVSA solution. On the contrary, the pure oil phase has a relatively low adhesion force of $45-50 \mu \mathrm{N}$ to the diamond tip, while the addition of vTA-CMp into the oil phase drastically decreases the adhesion force and correspondingly increased the viscosity. The viscosity was further confirmed by dynamic viscosity measurement (Figure $2 \mathrm{~d}$ ), revealing a viscosity of sunflower oil of $48 \mathrm{mPa} \cdot \mathrm{s}$ that was further enhanced to $60 \mathrm{mPa} \cdot \mathrm{s}$ by vTA$\mathrm{CMp}$ addition. One criterion to achieve a stable liquid object is the presence of a robust jammed interfacial film to retain the liquid shape. From this aspect, the increment in viscosity supports the interfacial strength of the final liquid object, although aqueous PVSA solution possesses a relatively low viscosity of $4 \mathrm{mPa} \cdot \mathrm{s}$.

The fluid resistance was further investigated for the vTACMp/oil and aqueous PVSA solution system to obtain the pressure drag force. As the load-displacement curve measured from the movement of the indenter during diamond tip immersion in the liquid indicates pressure drag force and slopes correspond to the fluid resistance, a steeper slope corresponds to denser material and higher resistance to flow (Figure S16). The addition of vTA-CMp to the oil phase decreased the pressure drag of the oil phase (Figure S17a), which is ascribed to the polarized structure of vTA-CMp. The formed electrostatically driven colloidal assembly leads to a repulsion of vTA-CMp nanosheets that results in decreased fluid resistance. On the contrary, aqueous PVSA solution possesses a relatively large pressure drag force (Figure S17b), which can be ascribed to the long polymer chain of PVSA, endowing the aqueous phase with enough "toughness" to maintain the interfacial jamming against collapses.

Subsequently, depth-sensing was attempted to study the interfacial layer structure, which is related to the forces experienced across the aqueous PVSA solution through the interfacial layer and finally to the vTA-CMp/oil phase (Figure $3 \mathrm{~d}$, dashed box and Figure $3 \mathrm{e}$ ). For investigation of the interfacial mechanical property in a changing $\mathrm{pH}$ environment and its effect on the configuration of liquid objects, a custommade cono-spherical glass tip (Figure S2) was used as a probe because of the intolerance of the conventional indentation tips with an acidic or basic environment. As the interfacial jamming is based on self-assembly on an anisotropic object, a nonuniform, all-round probing procedure was conducted (Figure S3). The gray curve in Figure $3 a-c$ is the fluid resistance of PVSA solution at the corresponding $\mathrm{pH}$ values, and a significant deviation from the PVSA loading path was found as the tip approached the interfacial area. For a $\mathrm{pH}$ value of 2, an obvious jump-in behavior, detected by an unstable and sharp load drop (Figure 3a, curve $a_{1}$, inset), was observed in $33 \%$ of the investigations (Figure 3a, pie chart, Figure S18a), indicating that the tip was immediately dragged into the oil phase which causes a sudden environment change. This suggests a weak protection of the interfacial film against external disruption, which is due to the relatively high interfacial tension between two phases that formed a mechanically weak interfacial area. As the PVSA chain is protonated to some extent at a $\mathrm{pH}$ value of $2,50 \%$ of pop-in behavior was detected with a stable and gentle load drop (Figure 3a, curve $a_{2}$, Figure $S 18 b$ ) when the tip interacted with the interface, which is usually caused by charged interaction between the tip and solution, indicating an elastic interfacial film. However, in $17 \%$ of the investigation (Figure $3 a$, curve $a_{3}$, Figure S18c), fluctuant and steady areas were found at the corner of the liquid object, which demonstrated the presence of well-assembled interfacial parts that can strongly protect against external probing. This might be ascribed to the nonuniform jamming of vTA-CMp colloids at the interfacial area and the enrichment of vTA-CMp at the interface. With lower interfacial tension between two phases at a $\mathrm{pH}$ value of 7, 33\% of jump-in was observed as well (Figure $3 b$, curve $b_{1}$, Figure $\mathrm{S} 19 \mathrm{a}$ ) in the investigation, which indicated that there is a weak interfacial area that is not strong enough to withstand the external stimulation, and the film was broken suddenly as the surrounding environment of the tip changed. A decreased number $(17 \%)$ of pop-in events (Figures $3 b, b_{2}$ and $S 19 b$ ) was observed compared to the $\mathrm{pH}=2$ environment, which is due to the increased deprotonation of the PVSA chain at the $\mathrm{pH}$ value of 7 , as less charged interactions between the tip and interface take place, and this also suggested less elasticity of the interfacial film than at $\mathrm{pH}$ 2. Importantly, for half of the investigated data points ( $50 \%$, Figures $3 \mathrm{~b}, \mathrm{~b}_{3}$ and $\mathrm{S} 19 \mathrm{c}$ ), a relatively smooth transition of resistance force during two phases was observed, which indicates a stable interaction between the interfacial elastic film and the external tip. The stable interaction suggests a strong mechanical characteristic of the interfacial film, resulting in a lower energy layer that leads to less resistance force for the tip to exceed. When the $\mathrm{pH}$ value of the solution reached 13 , no jump-in and pop-in events were observed during the probing, and a quite smooth transition period with no drop-in load was observed while the tip approached the interfacial area (Figures 3c and S20), which is because of the relatively small interfacial tension at the $\mathrm{pH}$ value of 13. As such, the interfacial energy needed to form a mechanically strong interfacial assembly was minimized. However, a steeper slope was detected while the tip indented, which suggests a significant extrusion of the oil from the " $\mathrm{H}$ " liquid object pattern in a basic environment $(\mathrm{pH}=13)$. In general, the indentation measurements showed that vTA-CMp is distributed non-uniformly around the liquid object, which is mainly due to the anisotropic nature of the liquid object.

In order to gain insights into the effect of $\mathrm{g}-\mathrm{C}_{3} \mathrm{~N}_{4}$ species on the structure formation and dispersibility in sunflower oil, another type of g- $\mathrm{C}_{3} \mathrm{~N}_{4}$ called $\mathrm{CM}$ (calcined by cyanuric acidmelamine as a precursor) was investigated. ${ }^{57} \mathrm{~A}$ dispersion of $\mathrm{CM} /$ oil with lower quality was obtained (Figure S21) that was utilized subsequently for molded liquid object formation. However, no identifiable shape was formed in aqueous PVSA solution, which might be due to the weak and disordered charge distribution of CM in oil dispersion (Figure S22a). The control experiment with $\mathrm{CM}$ showed the necessity of the altered charge structure in the liquid-liquid system. Hence, we assume that the formation of the liquid object is enabled by jamming of vTA-CMp at the interface, which effectively minimizes the interfacial tension and energy. Furthermore, the polar structure of vTA-CMp facilitates electrostatic interaction, with negatively charged PVSA leading to supramolecular bonding and formation of an elastic film that maintains the integrity and fidelity of the liquid object. Moreover, aqueous PVSA solution was substituted by CTAB and PSSA, respectively. No clear " $\mathrm{H}$ " shape was formed by filling with 

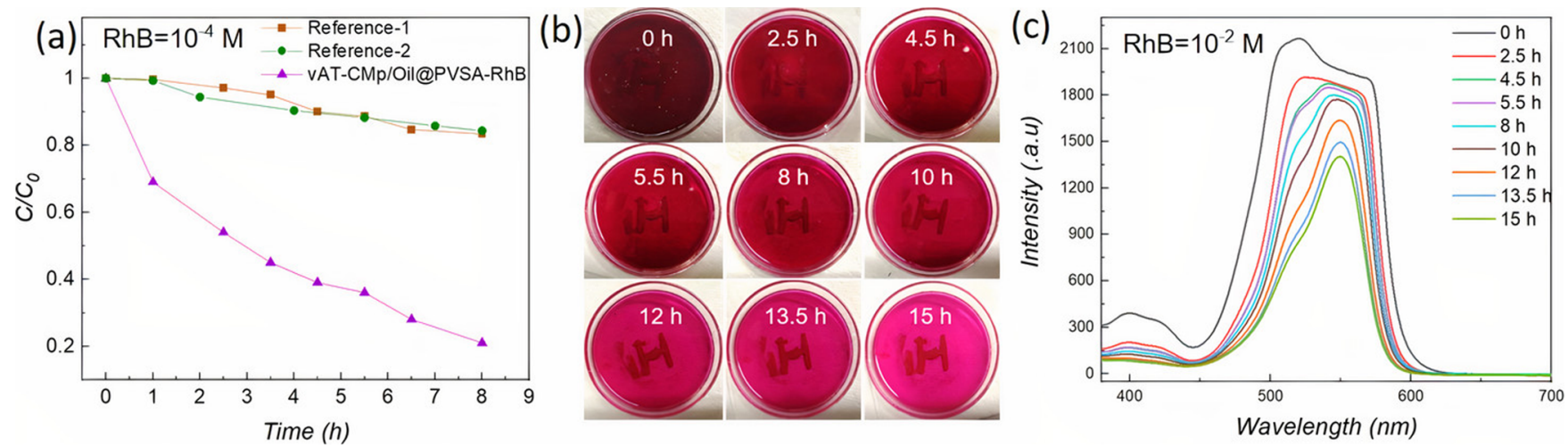

Figure 4. (a) Progress of photocatalytic degradation of PVSA-RhB $\left(10^{-4} \mathrm{M}\right)$ solution with a vTA-CMp/oil liquid structure under visible light irradiation. (Reference-1 is the photodegradation progress in PVSA-RhB solution, and reference- 2 is the photodegradation progress in RhB-PVSA solution extract from the vTA-CMp/oil@PVSA-RhB system). (b) Digital images of the liquid object formed from vTA-CMp/oil and aqueous PVSA solution in the presence of $\mathrm{RhB}\left(10^{-2} \mathrm{M}\right)$ under visible light irradiation after certain time intervals. and (c) Corresponding UV-vis absorption spectra of the solution at different time intervals related to (b).

vTA-CMp/oil in aqueous CTAB (Figure S22b), as the cationic surfactant $\mathrm{CTAB}$ is a small molecule that is not sufficient to form a stable interfacial layer with vTA-CMp. In the case of PSSA ( 30 wt \% in $\mathrm{H}_{2} \mathrm{O}$ ) (Figure S22c), a liquid object was formed in an incomplete and unstable state. A reason for this effect could be the different steric demand of both polymer types and chain flexibility. Furthermore, both polymer sidechains have different acidities. In comparison to small molecules, the $\mathrm{p} K_{\mathrm{a}}$ of benzenesulfonic acid is -2.8 and that of methanesulfonic acid is $-1.9,{ }^{58}$ which shows a difference of one order of magnitude. This further affects the polymer structure in water and the interaction with vTA-CMp, which causes slightly different liquid structures and less stability in the case of PSSA.

Liquid Object Photocatalysis. Formation of stable liquid objects is driven by charge interactions at the interface in the heterophase system, yet an additional advantage of vTA-CMp utilization is the photoactivity in the visible range (Figure S4d). These photocatalytic properties might be a direction for future applications in all-liquid biphasic photocatalysis processes. In order to study the interfacial dynamics and demonstrate the photoactivity of vTA-CMp, the liquid object was immersed in aqueous PVSA solution with $\mathrm{RhB}$ addition $\left(10^{-4} \mathrm{M}\right)$ (Figures $4 \mathrm{a}$ and S23a). Efficient photodegradation of $\mathrm{RhB}$ of $80 \%$ after $8 \mathrm{~h}$ irradiation was observed, which is in contrast to a reference sample (Figure $4 \mathrm{a}$, curve reference-1, Figure S23b) in the absence of a vTA-CMp/oil liquid object. Dye adsorption capacity of the structured liquid has been investigated with the same conditions in the dark, indicating that the saturated surface $\mathrm{RhB}$ adsorption on the liquid structure is about $30 \%$ (Figure S24). In addition, photodegradation was attempted in PVSA-RhB solution that was extracted from the vTA-CMp/oil@PVSA-RhB system. No obvious degradation performance was observed (Figure 4a, curve reference-2), which confirmed that the photodegradation ability of the vTA-CMp/oil@PVSA-RhB system did not result from dispersed vTA-CMp nanosheets in the aqueous phase but from the liquid object itself. In the so-formed liquid structures, vTA-CMp colloids have a role beyond charge interaction and stabilization, and their photocatalytic activity can be employed for further applications as well, therefore showing the potential application of liquid structures as encoding materials (photoactive liquid-liquid printed soft objects). In that sense, the object is concealed in the beginning with a dye, and it gradually appeared while the dye in the solution is photodegraded by liquid objects under visible light. The preformed liquid object is hardly detectable at the beginning because of the high $\mathrm{RhB}$ concentration $\left(10^{-2} \mathrm{M}\right)$, but gradual degradation of $\mathrm{RhB}$ via visible light reveals the original shape (Figure $4 \mathrm{~b}$ ). After $2.5 \mathrm{~h}$, the " $H$ " gradually appeared and was clearly observed after 4.5 h. The aqueous solution turned translucent after $12 \mathrm{~h}$ irradiation. The corresponding UV absorption spectra demonstrated a photodegradation of $40 \%$ after $15 \mathrm{~h}$ (Figure $4 \mathrm{c}$ ), yet it is important to mention that the $\mathrm{RhB}$ concentration used here $\left(10^{-2} \mathrm{M}\right)$ is almost two orders of magnitude higher than the common concentration used for photodegradation, 59 as high concentration is necessary for concealing the " $H$ " structure. This might be a reason for the relatively low photodegradation rate due to the significant light absorption of $\mathrm{RhB}$ in solution. Another obvious reason is that the interfacial area between vTA-CMp/oil and aqueous PVSA solution is rather low because of the macroscopic dimensions. Thus, only a limited amount of $\mathrm{RhB}$ molecules can reach the elastic film to undergo degradation (Figure S25). However, it is worth mentioning that the continuous aqueous phase shows significant mobility to enable interfacial photoinduced reactions on the jamming vTA-CMp/PVSA interfacial film with vTA-CMp as photoactive species.

\section{CONCLUSIONS}

In conclusion, an all-liquid molded object was constructed in an aqueous system by a versatile method, utilizing a colloidal dispersion of fluorescent vTA-CMp in edible sunflower oil as the inner phase and biocompatible aqueous PVSA solution as the outer phase. The jamming of vTA-CMp in cooperation with negatively charged sulfonate groups at the oil-water interface resulted in the formation of a liquid object by a supramolecular stabilized interface with $\mathrm{pH}$ tolerance in an acidic and alkaline environment. Interfacial tension and indentation were investigated to obtain insights on interfacial activity, properties of interfacial elastic films, and their formation mechanism. Furthermore, the photoactivity of vTA-CMp could be utilized to conceal and reveal the liquid object via photodegradation under visible light. Therefore, g$\mathrm{C}_{3} \mathrm{~N}_{4}$ appears to be a significant building block for liquid objects that might provide facile access to a variety of valuable applications, such as all-liquid biphasic photocatalysis, artificial 
compartmentalized systems, liquid-liquid printing, or bioprinting.

\section{ASSOCIATED CONTENT}

\section{SI Supporting Information}

The Supporting Information is available free of charge at https://pubs.acs.org/doi/10.1021/acsami.0c18064.

Synthesis methods of vTA-CMp, mold template, transmission electron microscopy, contact angle, UV-vis absorption, indentation, and pendant drop (PDF)

\section{AUTHOR INFORMATION}

\section{Corresponding Authors}

Baris Kumru - Department of Colloid Chemistry, Max Planck Institute of Colloids and Interfaces, 14476 Potsdam, Germany; (1) orcid.org/0000-0002-1203-4019;

Email: baris.kumru@mpikg.mpg.de

Bernhard V. K. J. Schmidt - Department of Colloid Chemistry, Max Planck Institute of Colloids and Interfaces, 14476 Potsdam, Germany; School of Chemistry, University of Glasgow, Glasgow G128QQ U.K.; 이이.org/0000-00023580-7053; Email: bernhard.schmidt@glasgow.ac.uk

\section{Authors \\ Qian Cao - Department of Colloid Chemistry, Max Planck Institute of Colloids and Interfaces, 14476 Potsdam, Germany \\ Shahrouz Amini - Department of Biomaterials, Max Planck Institute of Colloids and Interfaces, 14476 Potsdam, Germany}

Complete contact information is available at:

https://pubs.acs.org/10.1021/acsami.0c18064

\section{Funding}

Max Planck society.

Notes

The authors declare no competing financial interest.

\section{ACKNOWLEDGMENTS}

The authors acknowledge Tobias Schmidt for the fabrication of PA molds, Antje Völkel for density and viscosity measurements, and Marlies Gräwert for SEC measurements.

\section{REFERENCES}

(1) Cai, D.; Clegg, P. S. Stabilizing Bijels Using a Mixture of Fumed Silica Nanoparticles. Chem. Commun. 2015, 51, 16984-16987.

(2) Xie, G.; Forth, J.; Chai, Y.; Ashby, P. D.; Helms, B. A.; Russell, T. P. Compartmentalized, All-Aqueous Flow-Through-Coordinated Reaction Systems. chem 2019, 5, 2678-2690.

(3) Erni, P.; Jerri, H. A.; Wong, K.; Parker, A. Interfacial Viscoelasticity Controls Buckling, Wrinkling and Arrest in Emulsion Drops Undergoing Mass Transfer. Soft Matter 2012, 8, 6958-6967.

(4) Haraguchi, K. Development of Soft Nanocomposite Materials and Their Applications in Cell Culture and Tissue Engineering. J. Stem Cells Regen. Med. 2012, 8, 2.

(5) Vithani, K.; Goyanes, A.; Jannin, V.; Basit, A. W.; Gaisford, S.; Boyd, B. J. An Overview of 3D Printing Technologies for Soft Materials and Potential Opportunities for Lipid-based Drug Delivery Systems. Pharm. Res. 2019, 36, 4.

(6) Taylor, D. L.; in het Panhuis, M. Self-Healing Hydrogels. Adv. Mater. 2016, 28, 9060-9093.

(7) Yuan, T.; Cui, X.; Liu, X.; Qu, X.; Sun, J. Highly Tough, Stretchable, Self-Healing, and Recyclable Hydrogels Reinforced by in
Situ-Formed Polyelectrolyte Complex Nanoparticles. Macromolecules 2019, 52, 3141-3149.

(8) Mytnyk, S.; Olive, A. G. L.; Versluis, F.; Poolman, J. M.; Mendes, E.; Eelkema, R.; van Esch, J. H. Compartmentalizing Supramolecular Hydrogels Using Aqueous Multi-phase Systems. Angew. Chem., Int. Ed. 2017, 56, 14923-14927.

(9) Bayoumi, M.; Bayley, H.; Maglia, G.; Sapra, K. T. Multicompartment Encapsulation of Communicating Droplets and Droplet Networks in Hydrogel as a Model for Artificial Cells. Sci. Rep. 2017, 7, 45167.

(10) Cybulski, O.; Dygas, M.; Mikulak-Klucznik, B.; Siek, M.; Klucznik, T.; Choi, S. Y.; Mitchell, R. J.; Sobolev, Y. I.; Grzybowski, B. A. Concentric Liquid Reactors for Chemical Synthesis and Separation. Nature 2020, 586, 57-63.

(11) Akbulut, O.; Mace, C. R.; Martinez, R. V.; Kumar, A. A.; Nie, Z.; Patton, M. R.; Whitesides, G. M. Separation of Nanoparticles in Aqueous Multiphase Systems through Centrifugation. Nano Lett. 2012, 12, 4060-4064.

(12) Zeininger, L.; Nagelberg, S.; Harvey, K. S.; Savagatrup, S.; Herbert, M. B.; Yoshinaga, K.; Capobianco, J. A.; Kolle, M.; Swager, T. M. Rapid Detection of Salmonella enterica via Directional Emission from Carbohydrate-Functionalized Dynamic Double Emulsions. ACS Cent. Sci. 2019, 5, 789-795.

(13) Dinsmore, A. D.; Hsu, M. F.; Nikolaides, M. G.; Marquez, M.; Bausch, A. R.; Weitz, D. A. Colloidosomes: Selectively Permeable Capsules Composed of Colloidal Particles. Science 2002, 298, 10061009.

(14) Shi, S.; Russell, T. P. Nanoparticle Assembly at Liquid-liquid Interfaces: From the Nanoscale to Mesoscale. Adv. Mater. 2018, 30, 1800714 .

(15) Jeong, Y.; Chen, Y.-C.; Turksoy, M. K.; Rana, S.; Tonga, G. Y.; Creran, B.; Sanyal, A.; Crosby, A. J.; Rotello, V. M. Tunable Elastic Modulus of Nanoparticle Monolayer Films by Host-guest Chemistry. Adv. Mater. 2014, 26, 5056-5061.

(16) Liu, X.; Shi, S.; Li, Y.; Forth, J.; Wang, D.; Russell, T. P. Liquid Tubule Formation and Stabilization Using Cellulose Nanocrystal Surfactants. Angew. Chem., Int. Ed. 2017, 56, 12594-12598.

(17) Cui, M.; Emrick, T.; Russell, T. P. Stabilizing Liquid Drops in Nonequilibrium Shapes by The Interfacial Jamming of Nanoparticles. Science 2013, 342, 460-463.

(18) Xu, R.; Liu, T.; Sun, H.; Wang, B.; Shi, S.; Russell, T. P. Interfacial Assembly and Jamming of Polyelectrolyte Surfactants: A Simple Route To Print Liquids in Low-Viscosity Solution. ACS Appl. Mater. Interfaces 2020, 12, 18116-18122.

(19) Montelongo, Y.; Sikdar, D.; Ma, Y.; McIntosh, A. J. S.; Velleman, L.; Kucernak, A. R.; Edel, J. B.; Kornyshev, A. A. Electrotunable Nanoplasmonic Liquid Mirror. Nat. Mater. 2017, 16, $1127-1135$

(20) Sun, S.; Liu, T.; Shi, S.; Russell, T. P. Nanoparticle Surfactants and Structured Liquids. Colloid Polym. Sci. 2020, 1-14.

(21) Shi, S.; Liu, X.; Li, Y.; Wu, X.; Wang, D.; Forth, J.; Russell, T. P. Liquid Letters. Adv. Mater. 2018, 30, 1705800.

(22) Hou, H.; Li, J.; Li, X.; Forth, J.; Yin, J.; Jiang, X.; Helms, B. A.; Russell, T. P. Interfacial Activity of Amine-functionalized Polyhedral Oligomeric Silsesquioxanes (POSS): A Simple Strategy to Structure Liquids. Angew. Chem., Int. Ed. 2019, 58, 10142-10147.

(23) Sun, H.; Li, L.; Russell, T. P.; Shi, S. Photoresponsive Structured Liquids Enabled by Molecular Recognition at LiquidLiquid Interfaces. J. Am. Chem. Soc. 2020, 142, 8591-8595.

(24) Qian, B.; Shi, S.; Wang, H.; Russell, T. P. Reconfigurable Liquids Stabilized by DNA Surfactants. ACS Appl. Mater. Interfaces 2020, 12, 13551-13557.

(25) Gu, P. Y.; Chai, Y.; Hou, H.; Xie, G.; Jiang, Y.; Xu, Q. F.; Liu, F.; Ashby, P. D.; Lu, J. M.; Russell, T. P. Stabilizing Liquids Using Interfacial Supramolecular Polymerization. Angew. Chem., Int. Ed. 2019, 58, 12112-12116.

(26) Huang, C.; Sun, Z.; Cui, M.; Liu, F.; Helms, B. A.; Russell, T. P. Structured Liquids with $\mathrm{pH}$-Triggered Reconfigurability. Adv. Mater. 2016, 28, 6612-6618. 
(27) Jiang, Y.; Chakroun, R.; Gu, P.; Gröschel, A. H.; Russell, T. P. Soft Polymer Janus Nanoparticles at Liquid-Liquid Interfaces. Angew. Chem., Int. Ed. 2020, 59, 12751-12755.

(28) Feng, W.; Chai, Y.; Forth, J.; Ashby, P. D.; Russell, T. P.; Helms, B. A. Harnessing Liquid-in-liquid Printing and Micropatterned Substrates to Fabricate 3-Dimensional All-liquid Fluidic Devices. Nat. Commun. 2019, 10, 1095.

(29) Yang, W.; Godin, R.; Kasap, H.; Moss, B.; Dong, Y.; Hillman, S. A. J.; Steier, L.; Reisner, E.; Durrant, J. R. Electron Accumulation Induces Efficiency Bottleneck for Hydrogen Production in Carbon Nitride Photocatalysts. J. Am. Chem. Soc. 2019, 141, 11219-11229.

(30) Wang, X.; Maeda, K.; Thomas, A.; Takanabe, K.; Xin, G.; Carlsson, J. M.; Domen, K.; Antonietti, M. A Metal-free Polymeric Photocatalyst for Hydrogen Production from Water under Visible Light. Nat. Mater. 2009, 8, 76-80.

(31) Sun, J.; Schmidt, B. V. K. J.; Wang, X.; Shalom, M. Self-standing Carbon Nitride-based Hydrogels with High Photocatalytic Activity. ACS Appl. Mater. Interfaces 2017, 9, 2029-2034.

(32) Ong, W.-J.; Tan, L.-L.; Ng, Y. H.; Yong, S.-T.; Chai, S.-P. Graphitic Carbon Nitride $\left(\mathrm{g}-\mathrm{C}_{3} \mathrm{~N}_{4}\right)$-Based Photocatalysts for Artificial Photosynthesis and Environmental Remediation: Are We a Step Closer To Achieving Sustainability? Chem. Rev. 2016, 116, 71597329.

(33) Liao, G.; He, F.; Li, Q.; Zhong, L.; Zhao, R.; Che, H.; Gao, H.; Fang, B. Emerging Graphitic Carbon Nitride-based Materials for Biomedical Applications. Prog. Mater. Sci. 2020, 112, 100666.

(34) Barrio, J.; Volokh, M.; Shalom, M. Polymeric Carbon Nitrides and Related Metal-free Materials for Energy and Environmental Applications. J. Mater. Chem. A 2020, 8, 11075-11116.

(35) He, P.; Tang, X.; Chen, L.; Xie, P.; He, L.; Zhou, H.; Zhang, D.; Fan, T. Patterned Carbon Nitride-Based Hybrid Aerogel Membranes via 3D Printing for Broadband Solar Wastewater Remediation. Adv. Funct. Mater. 2018, 28, 1801121.

(36) Xia, P.; Zhu, B.; Yu, J.; Cao, S.; Jaroniec, M. Ultra-thin Nanosheet Assemblies of Graphitic Carbon Nitride for Enhanced Photocatalytic CO2 Reduction. J. Mater. Chem. A 2017, 5, 32303238.

(37) Li, A.; Cao, Q.; Zhou, G.; Schmidt, B. V. K. J.; Zhu, W.; Yuan, X.; Huo, H.; Gong, J.; Antonietti, M. Three-Phase Photocatalysis for the Enhanced Selectivity and Activity of $\mathrm{CO} 2$ Reduction on a Hydrophobic Surface. Angew. Chem., Int. Ed. 2019, 58, 14549-14555.

(38) Volokh, M.; Peng, G.; Barrio, J.; Shalom, M. Carbon Nitride Materials for Water Splitting Photoelectrochemical Cells. Angew. Chem., Int. Ed. 2019, 58, 6138-6151.

(39) Kim, J. K.; Park, S.; Yoo, R. J.; Jeong, H. J.; Oh, J.; Lee, Y. J.; Park, S.; Kim, D. W. Thin PEGylated Carbon Nitrides: WaterDispersible Organic Nanodots as Bioimaging Probes. Chem.-Eur. J. 2018, 24, 3506-3511.

(40) Kumru, B.; Barrio, J.; Zhang, J.; Antonietti, M.; Shalom, M.; Schmidt, B. V. K. J. Robust Carbon Nitride-based Thermoset Coatings for Surface Modification and Photochemistry. ACS Appl. Mater. Interfaces 2019, 11, 9462-9469.

(41) Fu, Q.; Ruan, Q.; McKenzie, T. G.; Reyhani, A.; Tang, J.; Qiao,

G. G. Development of a Robust PET-RAFT Polymerization Using Graphitic Carbon Nitride (g-C3N4). Macromolecules 2017, 50, 75097516.

(42) Kumru, B.; Shalom, M.; Antonietti, M.; Schmidt, B. V. K. J. Reinforced Hydrogels via Carbon Nitride Initiated Polymerization. Macromolecules 2017, 50, 1862-1869.

(43) Hu, C.; Lin, Y. R.; Yang, H. C. Recent Developments in Graphitic Carbon Nitride Based Hydrogels as Photocatalysts. ChemSusChem 2019, 12, 1794-1806.

(44) Kumru, B.; Antonietti, M. Colloidal Properties of the Metal-free Semiconductor Graphitic Carbon Nitride. Adv. Colloid Interface Sci. 2020, 283, 102229.

(45) Han, C.; Cui, Q.; Meng, P.; Waclawik, E. R.; Yang, H.; Xu, J. Direct Observation of Carbon Nitride-Stabilized Pickering Emulsions. Langmuir 2018, 34, 10135-10143.
(46) Xu, J.; Antonietti, M. The Performance of Nanoparticulate Graphitic Carbon Nitride as an Amphiphile. J. Am. Chem. Soc. 2017, $139,6026-6029$.

(47) Cao, Q.; Cui, Q.; Yang, Y.; Xu, J.; Han, C.; Li, L. Graphitic Carbon Nitride as a Distinct Solid Stabilizer for Emulsion Polymerization. Chem.-Eur. J. 2018, 24, 2286-2291.

(48) Cao, Q.; Heil, T.; Kumru, B.; Antonietti, M.; Schmidt, B. V. K. J. Visible-light Induced Emulsion Photopolymerization with Carbon Nitride as a Stabilizer and Photoinitiator. Polym. Chem. 2019, 10, 5315-5323.

(49) Kumru, B.; Antonietti, M.; Schmidt, B. V. K. J. Enhanced Dispersibility of Graphitic Carbon Nitride Particles in Aqueous and Organic Media via a One-pot Grafting Approach. Langmuir 2017, 33, 9897-9906.

(50) Yandrapalli, N.; Robinson, T.; Antonietti, M.; Kumru, B. Graphitic Carbon Nitride Stabilizers Meet Microfluidics: From Stable Emulsions to Photoinduced Synthesis of Hollow Polymer Spheres. Small 2020, 16, 2001180.

(51) Cao, Q.; Kumru, B.; Antonietti, M.; Schmidt, B. V. K. J. Graphitic Carbon Nitride and Polymers: A Mutual Combination for Advanced Properties. Mater. Horiz. 2020, 7, 762-786.

(52) Sun, J.; Phatake, R.; Azoulay, A.; Peng, G.; Han, C.; Barrio, J.; $\mathrm{Xu}$, J.; Wang, X.; Shalom, M. Covalent Functionalization of Carbon Nitride Frameworks through Cross-Coupling Reactions. Chem.-Eur. J. 2018, 24, 14921-14927.

(53) Kumru, B.; Cruz, D.; Heil, T.; Schmidt, B. V. K. J.; Antonietti, M. Electrostatic Stabilization of Carbon Nitride Colloids in Organic Solvents Enables Stable Dispersions and Transparent Homogeneous CN Films for Optoelectronics. J. Am. Chem. Soc. 2018, 140, 1753217537.

(54) Cui, Q.; Xu, J.; Wang, X.; Li, L.; Antonietti, M.; Shalom, M. Phenyl-Modified Carbon Nitride Quantum Dots with Distinct Photoluminescence Behavior. Angew. Chem., Int. Ed. 2016, 55, $3672-3676$

(55) Amini, S.; Kolle, S.; Petrone, L.; Ahanotu, O.; Sunny, S.; Sutanto, C. N.; Hoon, S.; Cohen, L.; Weaver, J. C.; Aizenberg, J.; Vogel, N.; Miserez, A. Preventing Mussel Adhesion using Lubricantinfused Materials. Science 2017, 357, 668-673.

(56) Kim, S. J.; Park, S. J.; Kim, S. I. Properties of Smart Hydrogels Composed of Polyacrylic acid/Poly (vinyl sulfonic acid) Responsive to External Stimuli. Smart Mater. Struct. 2004, 13, 317.

(57) Shalom, M.; Inal, S.; Fettkenhauer, C.; Neher, D.; Antonietti, M. Improving Carbon Nitride Photocatalysis by Supramolecular Preorganization of Monomers. J. Am. Chem. Soc. 2013, 135, 71187121.

(58) Guthrie, J. P. Hydrolysis of Esters of Oxy Acids: p K a Values for Strong Acids; Brønsted Relationship for Attack of Water at Methyl; Free Energies of Hydrolysis of Esters of Oxy Acids; and a Linear Relationship Between Free Energy of Hydrolysis and pK a Holding over a Range of 20 pK Units. Can. J. Chem. 1978, 56, 23422354.

(59) Wang, Y.; Wang, Y.; Chen, Y.; Yin, C.; Zuo, Y.; Cui, L.-F. Synthesis of Ti-doped Graphitic Carbon Nitride with Improved Photocatalytic Activity under Visible Light. Mater. Lett. 2015, 139, $70-72$. 